\title{
Utilidad de un nuevo y breve cuestionario para la depresión
}

The PHQ-9: validity of a brief depression severity measure. Kroenke K, Spitzer RL, Williams JB. J Gen Intern Med.2001 Sep;16(9):60613.

\section{Objetivo}

Evaluar la validez de un nuevo instrumento, el PHQ-9 (Patient Health Questionnaire 9), para evaluar la severidad de la depresión.

\section{Lugar}

Consultorios ambulatorios de medicina familiar, de medicina general, y de gineco-ostetricia, EE.UU.

\section{Evaluación del test diagnóstico}

EI PHQ-9 es el módulo de evaluación de depresión del PRIME-MD, una encuesta autoadministrada para evaluar problemas mentales frecuentes. Consta de nueve ítems que evalúan cada uno de los nueve criterios del DSM-IV en una escala que va desde 0 (para nada) hasta 3 (casi todos los días).

\section{Evaluación del test de referencia y otros resultados}

El diagnóstico de depresión se realizó a través de entrevistas psiquiátricas telefónicas estructuradas en una muestra de 580 pacientes a menos de dos días de completar el PHQ.Los entrevistadores estaban ciegos al mismo. La validez de construcción* se evaluó a través de la correlación con el SF-20, el ausentismo laboral, el número de visitas médicas, y la dificultad relacionada a los síntomas. La confiabilidad test-retest ${ }^{*}$ se evaluó comparando el resultado del PHQ-9 del paciente con el obtenido por el entrevistador.

\section{Resultados}

Se evaluaron 6000 pacientes en ocho consultorios de atención pri- maria y siete gineco-obstétricos. A medida que aumentaba la severidad evaluada por el PHQ-9, empeoraba el estado funcional de todos los dominios evaluados por el SF-20 (físico, social, mental, dolor, etc). También hubo mayor ausentismo, mayor número de consultas, más dificultad relacionada a los síntomas a medida que aumentaba la severidad evaluada por el PHQ-9.Los 580 pacientes en quienes se realizó la entrevista psiquiátrica no difirieron sociodemográficamente del resto. Tomando como punto de corte un puntaje $>=10$, la sensibilidad fue del $88 \%$ y la especificidad del $88 \%$ para el diagnóstico de depresión. En el caso de estar deprimidos, un puntaje de 5, 10, 15, 20 representaron una depresión leve, moderada, moderadamente severa y severa respectivamente. Los resultados fueron similares en los consultorios de medicina familiar/ general y los gineco-obstétricos. El área bajo la curva ROC fue de 0.95.la confiabilidad test-retest* fue muy buena $(0.84)$.

Tabla:Utilidad diagnóstica de distintos resultados

\begin{tabular}{|c|c|}
\hline Puntaje PHQ-9 & Coeficiente de Probabilidad* \\
$0-4$ & 0.04 \\
$5-9$ & 0.5 \\
$10-14$ & 2.6 \\
$15-19$ & 8.4 \\
$20-27$ & 36.8 \\
\hline
\end{tabular}

\section{Conclusiones}

EI PHQ-9 fue útil no solo para diagnosticar depresión, sino que resultó confiable y válido también para la evaluación de su severidad.

Fuente de Financiamiento:laboratorio PFIZER (que financió el desarrollo del PRIME MD)

\section{Comentario}

La depresión no es solo un problema de salud frecuente en atención primaria, sino que también es subdiagnosticado y subtratado. ${ }^{1}$ Recientes recomendaciones de la fuerza de tareas preventivas de EE.UU (USPSTF) concluye que el rastreo de depresión se asocia a mejores resultados clínicos ${ }^{2}$.Meta-análisis sugieren que el rastreo y feedback reducen el riesgo de depresión persistente (RR 0.87 [IC95\% 0.79 a 0.95]), más aun si es utilizado conjuntamente con cambios en el sistema de salud para ayudar al tratamiento y seguimiento2. La recomendación para rastreo en adultos es tipo $\mathrm{B}^{*}$ (la evidencia es insuficiente en niños y adolescentes[Tipo $\left.\left.\mathrm{I}^{*}\right]\right)^{2}$.

En este estudio el PHQ-9 demostró ser válida y confiable no solo para el diagnóstico de depresión sino para evaluar su severidad. También parece ser fácil de administrar y buena como ayudamemoria de los nueve criterios diagnósticos del DSM-IV para depresión mayor (se diagnostica depresión mayor si se encuentran 5 de los nueve síntomas en las últimas dos semanas, y por lo menos uno de ellos es ánimo deprimido o anhedonia ).También es de hacer notar que existen muchos otros cuestionarios para diagnóstico de depresión con similares características, y algunos de ellos fueron validados en español en la Argentina (hasta donde yo tengo conocimiento, la escala de depresión geriátrica GDS2 y el inventario de Beck ${ }^{3}$ ). Es importante señalar que antes de adoptar un instrumento para la práctica, el mismo debe ser validado en el idioma y la población en que uno trabaja.

Conclusión del comentario: A pesar de que el PHQ-9 parece un instrumento promisorio, tanto como herramienta de diagnóstico y para evaluar la severidad, debemos esperar su validación al español para poder llevarla al consultorio.Lo que queda claro es que, más allá del instrumento elegido, el rastreo de depresión parece conferir beneficios importantes a los pacientes, si uno hace algo con el resultado del test.

\section{Dr. Federico Augustovski [ Unidad de Medicina Familiar y Preventiva. Hospital Italiano de Buenos Aires ]}

${ }^{*}$ Ver glosario

PHQ-9 (traducción tentativa del comentador)

¿Durante las últimas dos semanas, cuan frecuentemente le han causado alguna molestia algunos de los siguientes problemas? (todas se contestan 0 [para nada]; 1 [varios días]; 2 [más de la mitad de los días];3 [casi todos los días].Luego se suman los puntos (puntaje

posible 0 a 27 ).
1.Poco interés o placer en hacer cosas

1.Poco interés o placer en hacer cosas

3.Problemas para dormirse o mantener el sueño;o dormir demasiado
4.Sentirse cansado o con poca energía

5.Poco apetito o comer demás

6.Sentirse mal consigo mismo - o que usted es una falla o una decepción para usted o su familia.

7.Problemas para concentrarse en las cosas, como leer el diario o mirar televisión.

8. ¿ Hablar o moverse tan lentamente que otras personas lo notan? O lo contrario, estar tan agitado o inquieto que estuvo en movimiento mucho más de lo habitual.

9. Pensamientos acerca de que estaría mejor si se muriera o lastimara de alguna manera.

\section{Referencias}

1.Botargues, M.Razé, A.Ansiedad, Depresión e Insomnio. En:PROFAM:Programa de Educación a Distancia de Medicina Familiar y Ambulatoria / Esteban Rubinstein, dir. Buenos Aires: Hospital Italiano de Buenos Aires. Departamento de Docencia e Investigación, c1998.v.6, p.213-253.

2.Screening for depression:recommendations and rationale.Ann Intern Med.2002 May 21;136(10):760-4.

3.Carrete P, Augustovski F, Gimpel N, Fernandez S, Di Paolo R, Schaffer I, Rubinstein F.V alidation of a telephone-administered geriatric depression scale in a hispanic elderly population. J Gen Intern Med.2001 Jul;16(7):446-50.

4.Bonicatto S, Dew AM, Soria JJ. Analysis of the psychometric properties of the Spanish version of the Beck Depression Inventory in Argentina. Psychiatry Res 1998 Jul 13;79(3):277-85 